\title{
Enhanced EISCAT UHF backscatter during high-energy auroral electron precipitation
}

\author{
N. M. Schlatter ${ }^{1}$, N. Ivchenko ${ }^{1}$, T. Sergienko ${ }^{2}$, B. Gustavsson ${ }^{3}$, and B. U. E. Brändström ${ }^{2}$ \\ ${ }^{1}$ School of Electrical Engineering, Royal Institute of Technology, Teknikringen 31, 10044 Stockholm, Sweden \\ ${ }^{2}$ Institute for Space Physics, Rymdcampus 1, 98192 Kiruna, Sweden \\ ${ }^{3}$ EISCAT scientific association, Rymdcampus 1, 98192 Kiruna, Sweden. Now at: Department of Physics and Technology, \\ University of Troms $\varnothing, 9037$ Troms $\varnothing$, Norway
}

Correspondence to: N. M. Schlatter (nmsc@kth.se)

Received: 21 January 2013 - Revised: 11 July 2013 - Accepted: 3 September 2013 - Published: 9 October 2013

\begin{abstract}
Natural enhancements in the backscattered power of incoherent scatter radars up to 5 orders of magnitudes above the thermal backscatter are sometimes observed at high latitudes. Recently observations of enhancements in the backscattered power including a feature at zero Doppler shift have been reported. These enhancements are limited in altitude to tens of kilometers. The zero Doppler shift feature has been interpreted as a signature of electron density cavitation. Enhanced plasma lines during these observations have also been reported. We report on the first EISCAT UHF observations of enhanced backscattered radar power including a zero Doppler shift feature. The enhancements originated from two distinct and intermittent layers at about $200 \mathrm{~km}$ altitude. The altitude extent of the enhancements, observed during auroral high-energy electron precipitation, was $<2 \mathrm{~km}$.
\end{abstract}

Keywords. Ionosphere (Auroral ionosphere)

\section{Introduction}

Incoherent scatter radars (ISR) measure bulk properties of ionospheric plasma predominantly by analysis of the spectral power backscattered from thermal ion acoustic waves. At high latitudes the spectral power in the ion acoustic regime is occasionally enhanced above the thermal backscatter. These so-called naturally enhanced ion acoustic lines (NEIAL) occur in the altitude range of 140 to $1900 \mathrm{~km}$ during magnetically disturbed conditions (Foster et al., 1988; Rietveld et al., 1991; Sedgemore-Schulthess and St. Maurice, 2001; Ogawa et al., 2006, 2011). The enhancements observed during NEIALs can be up to 4-5 orders of magnitude and are typically extended over hundreds of kilometers in altitude.

Observations of NEIALs with a radar pointing along the direction of the magnetic field have been reported since the 1980s (e.g. Rietveld et al., 1991) and have been related to intense red auroral arcs using all-sky cameras (Collis et al., 1991). More recent reports using high time resolution (0.2 s) and the two dishes of the EISCAT Svalbard Radar (ESR; $500 \mathrm{MHz}$ ) as interferometer showed that NEIALs are very short lived and that NEIALs are limited to a few hundred meters in the plane perpendicular to the magnetic field (Grydeland et al., 2003; Grydeland et al., 2004). Using narrow field of view cameras Blixt et al. (2005) presented observations indicating that auroral rays and the region of enhanced backscatter occur on the same field line.

NEIALs of a different type observed with the EISCAT VHF radar $(224 \mathrm{MHz})$ have been reported by Rietveld et al. (2002). The observations show NEIAL with a feature at zero Doppler shift from a limited-altitude region and simultaneously enhanced plasma lines (see also Isham et al., 2012). Geometrical effects need to be considered for these observations as the VHF radar was pointing vertically during their experiment. Similar observations were reported on using the ESR (Strømme et al., 2005). Ekeberg et al. (2012) conducted a statistical study of these type of NEIALs based on one year of data taken with the ESR during the International Polar Year and found 48 events occurring during magnetically disturbed conditions with the peak of occurrence in the premidnight sector. The altitude of the enhancements is close to the $\mathrm{F}$ region peak. A distinct zero Doppler shift feature has previously been reported by Forme et al. (1995) using the 
EISCAT VHF radar, but due to possible instrumental effects the central feature has not been regarded further.

Michell and Samara (2010) have reported on NEIALs observations with the Poker Flat Incoherent Scatter Radar (PFISR; $449 \mathrm{MHz}$ ) from a limited-altitude region with a zero Doppler shift feature. Simultaneous measurements with narrow field of view imagers relate the observations to boundaries of auroral precipitation and filamentary structures. Furthermore Akbari et al. (2012) reported on NEIALs observed with the PFISR from a limited-altitude region with a zero Doppler shift feature and enhanced plasma lines at two frequencies. The altitude of these enhancements is close to $250 \mathrm{~km}$ and the altitude extent, while unresolved, well below $70 \mathrm{~km}$.

A number of theories have been proposed to explain NEIALs which can be grouped into either streaming instabilities - where streaming occurs between thermal ions or electrons, different ion species and thermal electrons - directly exciting ion acoustic waves or excitation of ion acoustic waves by Langmuir turbulence (e.g. Rietveld et al., 1991; Forme, 1999; Sedgemore-Schulthess and St. Maurice, 2001; Isham et al., 2012). Another model recently proposed by Ekeberg et al. (2010) describes NEIAL observations from limited-altitude regions with almost flat ion line spectra, i.e. spectrally uniform radar power enhancements, caused by increased incoherent backscatter cross section driven by ionsolitary waves. Bahcivan and Cosgrove (2008) proposed a model for NEIALs based on observations of ion cyclotron waves in the vicinity of arcs with strong electric fields observed with the FAST satellite. In this model the ion acoustic instability is driven by the ion cyclotron waves.

Excitation of enhanced ion acoustic waves by enhanced Langmuir waves is described in Langmuir turbulence (LT) theory (e.g. Robinson, 1997). The LT description can be divided into the weak Langmuir turbulence (WLT) and the strong Langmuir turbulence (SLT) regime distinguished by the energy available in the driver. While SLT involves phasecoherent wave interactions, Langmuir waves are described by their intensity in the WLT approximation and phase relations are unimportant. LT in the ionosphere has been numerically simulated using a WLT approach (Pavan et al., 2010; Ziebell et al., 2011; Sullivan et al., 2008) with a low-energy electron beam driver. In the WLT approach waves are described by their kinetic equations and random wave phases are assumed. Guio and Forme (2006) use a SLT approach and simulate the full Zakharov (Zakharov, 1972) set of equations with a low-energy electron beam driver. In the SLT approach density cavities (cavitons) are formed by a ponderomotive force due to the Langmuir oscillations. These cavitons are a possible scatter source for a zero Doppler shift feature in ISR measurements (DuBois et al., 1991). For ISRs to measure the coherent backscatter from cavitons, the quasiperiodic cavitons must have a separation $(L)$ of about the radar Bragg wavelength. The spatially averaged wave energy density necessary to create the cavitons is proportional to $1 / L^{2}$ (Robinson, 1997; Guio and Forme, 2006).

In this paper we present EISCAT UHF $(930 \mathrm{MHz})$ backscatter enhancements from the F region. The enhanced backscattered radar power is observed during the transit of a slow moving arc with high-energy electron precipitation. While NEIALs are commonly observed during dynamical aurora, the aurora prevailing during this event is stable over several minutes. The herein reported observations comprise the first reported observations of a zero Doppler shift feature measured with the EISCAT UHF radar during natural conditions. In Sect. 2 the observations are presented and in Sect. 3 we discuss the observations with respect to current theories of NEIALs.

\section{Observations and analysis}

The EISCAT UHF radar at Ramfjorden, Norway was operated on 12 December 2006, using the radar program arcl. Data were collected in the direction of magnetic zenith corresponding to an elevation of $77.5^{\circ}$ and azimuth of $185.1^{\circ}$. This radar program uses an alternating code with 64 bits, each $6 \mu$ s long. Altitudes between 96 and $422 \mathrm{~km}$ are covered. The advantage of this radar program is the high time and range resolution of $0.44 \mathrm{~s}$ and $0.9 \mathrm{~km}$, respectively. For the time of interest no optical data were available from imagers at the EISCAT site due to weather conditions. Optical ground-based data however are available from two ALIS (Steen et al., 1997) stations located at Skibotn, Norway and Kiruna, Sweden after 17:00 UT.

An auroral arc passed through the radar beam, producing enhanced $\mathrm{E}$ region ionisation for about $20 \mathrm{~min}$ around 17:00 UT, see Fig. 1. During the event, electron densities at $100 \mathrm{~km}$ exceeded $10^{12} \mathrm{~m}^{-3}$. The $\mathrm{F}$ region electron density above $200 \mathrm{~km}$ altitude increased throughout the event. After $\sim$ 17:00 UT the temporal variability of the E region electron density increases. The ion production rate profile depends on the energy distribution of the electron precipitation. The peak of the ionisation can be related to the predominant energies (e.g. Rees , 1963). The observed peak altitude of roughly $100 \mathrm{~km}$ corresponds to characteristic energies of $10-20 \mathrm{keV}$.

Images from Skibotn, about $50 \mathrm{~km}$ east/south-east of the radar, show a single arc located within the radar beam for the period of the event. Four images taken at approx. 17:02, 17:03, 17:04, and 17:08 UT are shown in the bottom row of Fig. 2. Partial cloud coverage reduced the quality of the optical measurements. The optical data, however, clearly indicate an auroral configuration stable on large-scale sizes over the time period of the event.

The measured backscattered radar power, plotted in the three upper panels of Fig. 2, shows two thin layers between 180 and $230 \mathrm{~km}$ altitude with intermittent enhancements, starting at about 16:59 UT and lasting until the end of the 

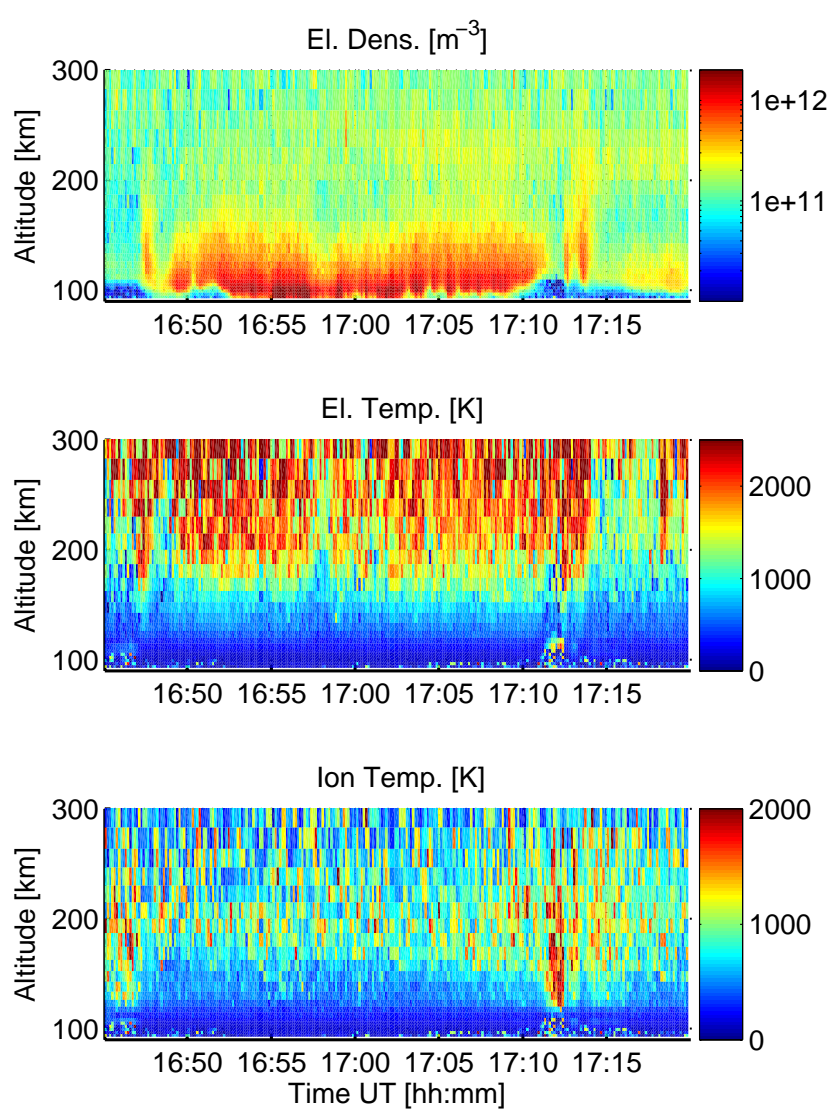

Fig. 1. Overview of the radar data for the event on 12 December 2006. The three panels show electron density, electron temperature and ion temperature respectively.

precipitation at 17:11 UT. The enhancements in the backscattered power reached up to almost two orders of magnitude.

The alternating radar codes rely on the target being stable over the time of the transmission of the whole code sequence, in the case of the arc1 experiment corresponding to the data slice of $0.44 \mathrm{~s}$. While some of the enhancements were properly decoded and observed in several consecutive slices, some slices exhibit sporadic enhancements over the whole length of the coded pulse, indicating considerable variation of the scattering cross section over the slice time. The power of a single layer was typically spread over two range gates corresponding to $1.8 \mathrm{~km}$.

Both layers were in the lower F region. The top layer altitude was around $220 \mathrm{~km}$ before 17:05 UT, reducing to 205$210 \mathrm{~km}$ at 17:08 UT. While the upper layer was observed during most of the enhancements, the lower layer was most prominent between 17:00 and 17:05 UT, only seen occasionally at later time. Between 17:02 and 17:04 UT the altitude of the layer increases from $180 \mathrm{~km}$ to nearly $200 \mathrm{~km}$, later gradually reducing to $190 \mathrm{~km}$.

The radar spectra for the power enhancements show enhanced ion line shoulders as well as a strong zero Doppler shift feature. In Fig. 3 two examples for enhanced backscatter spectra are shown. The feature at zero Doppler shift was typically stronger in power than the ion line shoulders for the observations.

The enhancements in the $\mathrm{F}$ region were observed during periods of intense electron precipitation. In Fig. 2c, the peak backscattered power from ranges between 180 and $246 \mathrm{~km}$ (gray line) is plotted together with the mean backscattered power from ranges between 96 and $117 \mathrm{~km}$ (black line). The peak backscattered power is an indicator of the enhancement present in the lower $\mathrm{F}$ region. The mean backscatter power is related to the electron concentration in the $\mathrm{E}$ region, and is related to the electron precipitation. While the optical arc is observed to be persistent and vary only moderately in intensity over the observation period, the E region ionisation observed by the radar is more bursty. On short timescales, the electron concentration in the $\mathrm{E}$ region grows during a precipitation burst until an equilibrium is reached, and decays when the precipitation ceases. Thus, time increase or constant high level of electron density corresponds to precipitation bursts. The plot indicates a pronounced relation between the precipitation and the power enhancements. The pronounced relation between the precipitation and the power enhancements is seen more clearly in the shorter time interval plotted in Fig. 2d.

Figure 4 shows the electron density estimated by standard analysis (Lehtinen and Huuskonen, 1996) for $16 \mathrm{~s}$ and $16.2 \mathrm{~km}$ integrated data for both the upper and lower layer of enhancement. While the layers changed in altitude in a non-monotonic way, the electron density at the enhancement altitudes grows throughout the event, from $1-1.5 \times 10^{11} \mathrm{~m}^{3}$ to over $2 \times 10^{11}$ by 17:09 UT. When both enhanced layers are observed, the density at both locations have very similar values.

To investigate the relative location of the enhancements with respect to the density profile, and specifically to the F region peak in more detail, a conditionally averaged background electron density profile was constructed as follows. In the first step, power profiles averaged over a $16 \mathrm{~s}$ and $0.9 \mathrm{~km}$ window were used to determine the altitude of the enhancements. In a second step the same set of $16 \mathrm{~s}$ time averaged data were used to identify data without enhancements, preceded or followed by data with an enhancement in the top layer. Thus, the altitude of the top enhancement is known from one of the neighboring data dumps. An average power profile referred to the altitude of the top layer enhancement was then constructed from the non-enhanced dumps and is plotted as a black line in the top panel of Fig. 5. A third order polynomial, dashed black line, was fitted to the power profile where data points within $\pm 4.5 \mathrm{~km}$ altitude of the upper enhancement were omitted. The constructed power profile is almost flat at the altitude of the enhancements with an increase towards lower altitudes. The electron density profile was estimated by correcting the obtained power profile for the electron to ion temperature ratio and is shown in the bottom panel 


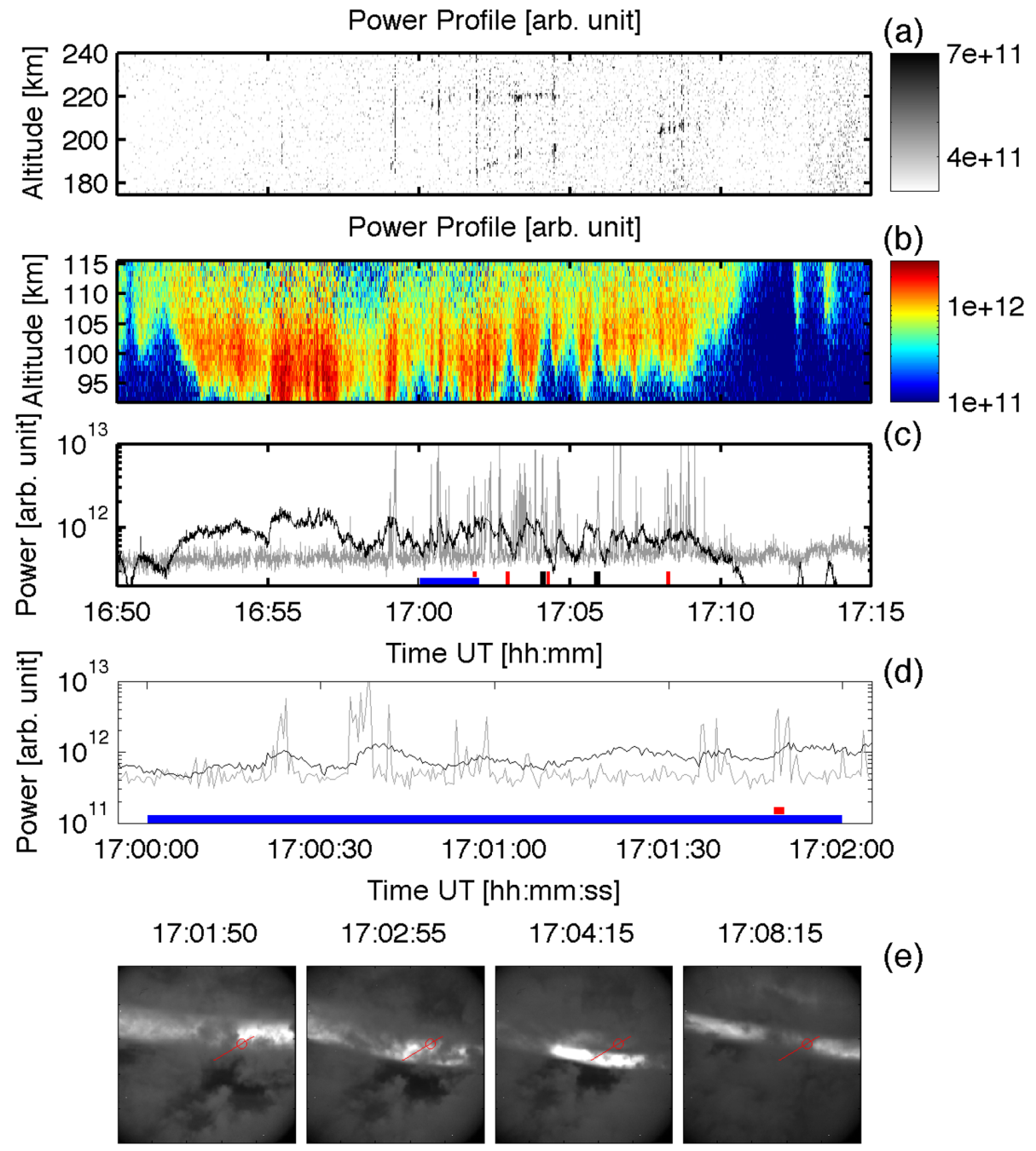

Fig. 2. Backscattered radar power from the lower F region and the lower E region are shown in (a) and (b) respectively. (c) Shows the mean backscattered power from these two regions, where the black line is the mean for the lower E region and the gray line the peak power from F region. The blue marker indicates the detail shown in (d). (e) Four images taken with the Skibotn ALIS camera are shown. The red line indicates the radar beam from 90 to $250 \mathrm{~km}$ with the red circle corresponding to an altitude of $100 \mathrm{~km}$. Indicators in (c) and (d) mark times of shown optical data (red) and radar spectra (black).

of Fig. 5 as a black dashed line. The electron density profile derived by standard data analysis (Lehtinen and Huuskonen, 1996) with time integration from 17:00-17:06 UT (16:5216:58 UT) is shown as a black line (grey line).

The peak in electron density (while not very pronounced) is observed at $220 \mathrm{~km}$ in the standard analysis and at $240 \mathrm{~km}$ in the electron density profiles excluding the power enhancements. The analysis confirms that the top layer with enhanced power is observed at or just below the F region peak, while the lower layer is observed on a slope of the density profile as it increases downwards towards the $\mathrm{E}$ region. Notably, no $F$ region peak is present in the integration of 16:5216:58 UT, prior to the period when the enhancement layers were observed.

\section{Discussion}

Observations of NEIALs were reported using the EISCAT VHF radar (224 MHz), the EISCAT UHF radar $(930 \mathrm{MHz})$, the ESR $(500 \mathrm{MHz})$ and the PFISR $(449 \mathrm{MHz})$. No observations of NEIALs with the Sondrestrom incoherent scatter radar $(1280 \mathrm{MHz})$ are known to the authors. A naturally excited zero Doppler shift feature has previously been reported in observations with the EISCAT VHF radar, ESR and PFISR.

Among the models for NEIALs, the Langmuir turbulence model and the model proposed by Ekeberg et al. (2010) include enhanced radar backscatter at zero Doppler shift. While in some observations of NEIALs the enhanced radar backscatter spectra are nearly spectrally uniform in between 

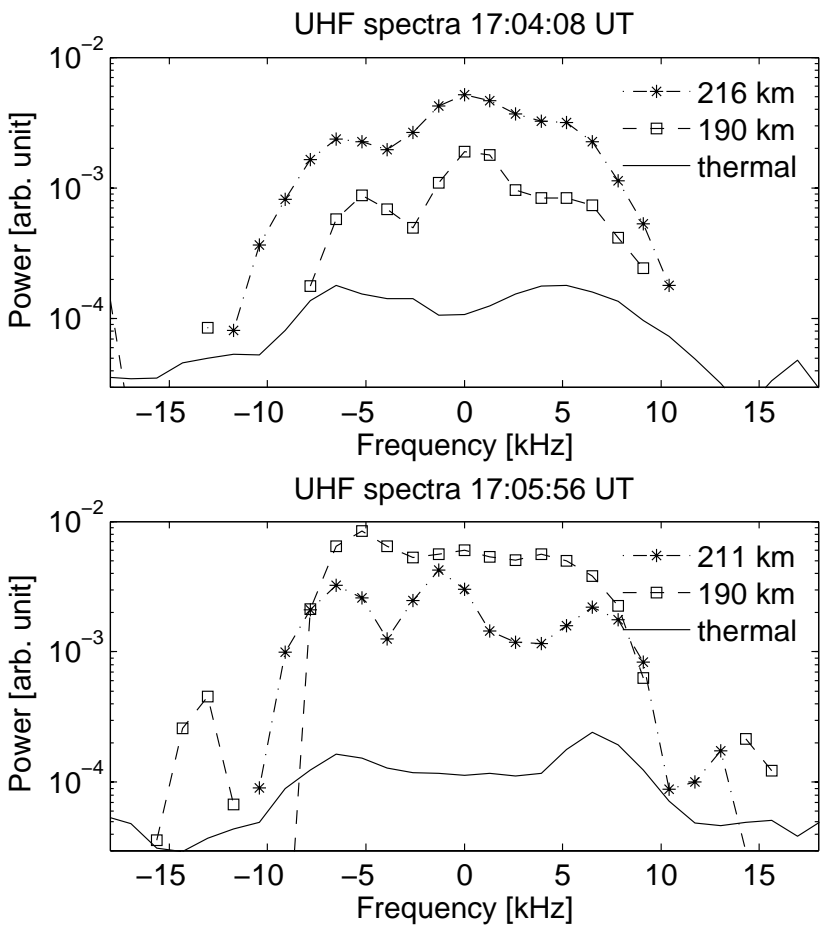

Fig. 3. Radar ion line spectra at 17:04:08 and 17:05:56 UT for the upper (dot-dashed line, asterisks) and lower (dashed line, squares) enhancements with $4 \mathrm{~s}$ integration time. The two solid lines show thermal spectra with integration time of $20 \mathrm{~s}$ from the altitude region in between the two layers of enhanced backscattered power.

the enhanced ion line shoulders (Michell et al., 2008; Michell and Samara, 2010; Ekeberg et al., 2010; Akbari et al., 2012), the enhanced radar spectra reported herein and those reported by Isham et al. (2012) show a distinct zero Doppler shift feature. A distinct zero Doppler shift feature is only accounted for in LT models.

In the LT model the spatially averaged wave energy density necessary to create cavitons is proportional to $1 / L^{2}$ (Robinson, 1997; Guio and Forme, 2006). To observe enhanced radar power backscattered from cavitons, the spacing $L$ of the cavitons needs to be close to half the radar wavelength. For observations with the EISCAT UHF radar the wavelength is $\sim 0.32 \mathrm{~m}$. Langmuir waves can be excited by the bump-in-tail instability when the resonance condition is fulfilled, i.e. $\omega_{\mathrm{L}} \approx k_{\mathrm{L}} v_{\mathrm{b}}$ where $\omega_{\mathrm{L}}$ and $k_{\mathrm{L}}$ are the wave frequency and vector of the excited Langmuir wave respectively and $v_{\mathrm{b}}$ the velocity of the beam electrons. LT describes the decay of primary excited Langmuir waves into daughter Langmuir waves and ion acoustic waves. The wave vector of the excited ion acoustic waves is $\approx 2 k_{\mathrm{L}}$ and enhanced ion line backscatter is observed when the radar Bragg condition of the radar is fulfilled. The energy of an electron beam exciting Langmuir waves and via LT subsequently ion acoustic waves, observed with the UHF radar, is necessarily low, of the order of a few $\mathrm{eV}$. The energy of precipitating electrons

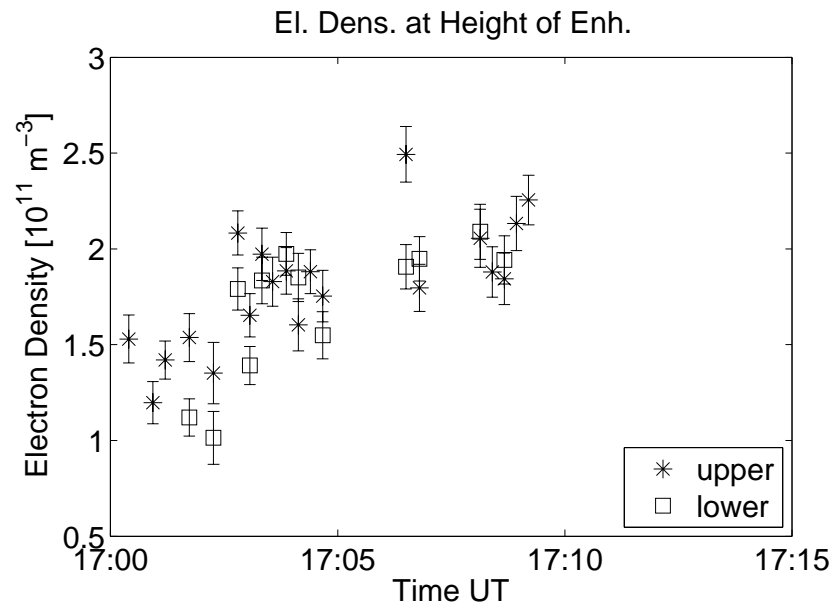

Fig. 4. The electron densities at the altitude of the observed enhancements in backscattered radar power are shown, where asterisks correspond to the upper layer and squares to the lower layer. The mean electron density is $1.76 \times 10^{11} \mathrm{~m}^{-3}$. Integration of $4 \mathrm{~s}$ was used.
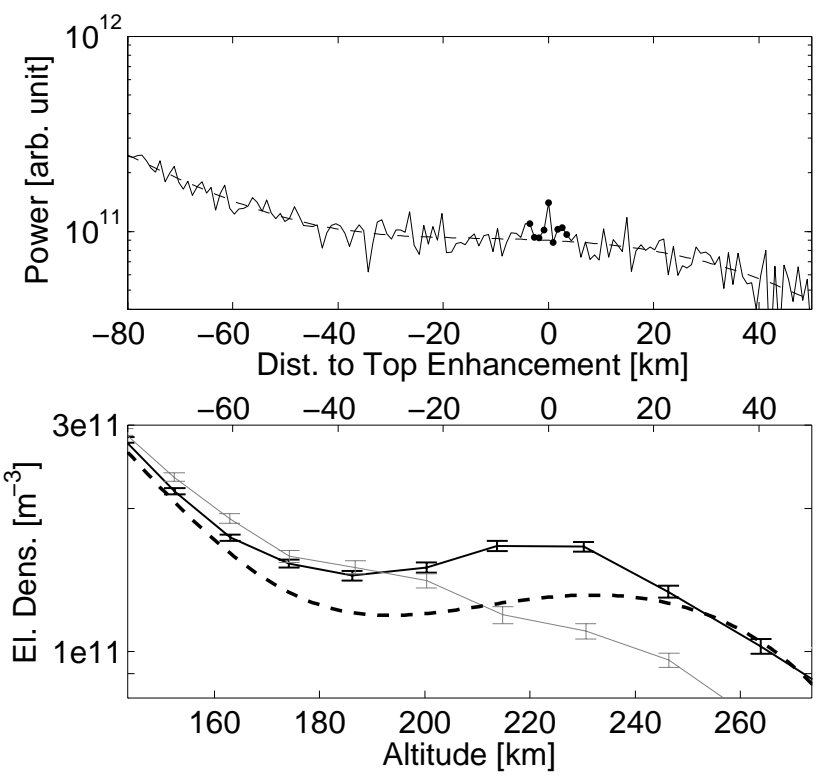

Fig. 5. The top panel shows the power profile with conditional integration (described in the text) between 1700 and 1706 (solid line). A polynomial fit is shown as dashed line. The lower panel shows the power profile in the top panel, adjusted to account for the measured electron-to-ion temperature ratio (dashed line). Electron density profile obtained by ion line fitting (standard analysis) with time integration 1700-1706 (1652-1658) is shown as solid black (gray) line with error bars. The curves are aligned such that the distance to the top enhancement of the temperature corrected power profile is relative to an altitude of $223.5 \mathrm{~km}$. 
in the event is clearly a few $\mathrm{keV}$. Thus, the electron population giving rise to LT and enhanced UHF backscatter is possibly caused by a secondary process in the ionosphere. A source of parallel motion of thermal electrons and ions are electric fields owing to charge accumulation due to auroral precipitation.

The altitude extent of regions with enhanced backscattered radar power is limited to about $2 \mathrm{~km}$ in our observations. While unresolved, the altitude extent has been reported to be $\sim 10 \mathrm{~km}$ (Isham et al., 2012) and $72 \mathrm{~km}$ (Akbari et al., 2012; Michell and Samara, 2010; Michell et al., 2008) in recent observations of a enhanced zero Doppler shift features. More common observations of NEIALs, where no zero Doppler shift feature is observed, typically extend over hundreds of kilometers and have been related to dynamic aurora exhibiting fine-scale structuring. The electron acceleration in this type of aurora is thought to be driven by dispersive Alfén waves. Dispersive Alfén waves drive suprathermal electron bursts with a wide range of energies of accelerated electrons. Thus, if interpreted in terms of weak Langmuir turbulence driven by a bump-in-tail instability, a wide range of primary Langmuir waves is excited and the matching condition for an ISR is likely to be fulfilled. The large-scale structure of the aurora underlying the reported event is stable over several minutes. In the context of Langmuir turbulence the small altitude extent of the enhanced backscatter regions suggests excitation of a narrow band of primary Langmuir waves. The small altitude extent of the enhancement occurring on a relatively weak altitude gradient in background electron density is surprising, and to our understanding, difficult to reconcile with existing models for NEIALs.

Ziebell et al. (2011) simulate a beam-driven WLT within the auroral density cavity (Calvert, 1981). Their simulations indicate that an ISR with suitable wave vector could observe NEIALs from two distinct altitude regions where the matching condition with the enhanced ion acoustic fluctuations is fulfilled. Similarly, in our observations the regions of enhanced radar backscatter are observed close to an apparent shallow density cavity. However, for cavitating turbulence a wide range of $k$ values are excited. Thus, the matching condition with the radar would be expected to be fulfilled over a wider altitude range.

The auroral arc present during the event is accompanied by anomalous heating (e.g. Buchert et al., 2008) at the poleward edge of the arc passing the radar beam around 17:12 UT. Electron temperatures at $110 \mathrm{~km}$ altitude reach up to $2000 \mathrm{~K}$. The anomalous heating and the high ion temperatures indicate the presence of strong electric fields in the vicinity of the arc. Observations by Michell and Samara (2010) and Ekeberg et al. (2010) indicate the presence of high electric fields during the reported events as well. Bahcivan and Cosgrove (2008) suggested a mechanism for ion cyclotron waves, in the vicinity of arcs with strong electric fields and shear, to excite enhanced levels of ion acoustic fluctuations and produce enhanced backscatter in incoherent scatter experiments.
A zero Doppler shift feature as observed in our ISR measurements during NEIALs is however not predicted by their model.

One can wonder why power enhancements with small altitude extent have not been reported previous to Rietveld et al. (2002). Non-thermal enhancements of the backscattered power will result in overestimated electron densities in analysis of incoherent scatter data. However, due to the commonly used and necessary altitude and time resolution of incoherent scatter data, these power enhancements from limited-altitude regions remain unnoticed. We find in our observations that the difference between results obtained with standard ISR analysis tools and estimates of the electron density profile derived by conditional averaging over power profiles is little.

\section{Conclusions}

We presented EISCAT UHF observations of naturally enhanced ion acoustic lines from two distinct altitude regions during auroral high-energy electron precipitation. Spectral features of the enhancements show a distinct feature at zero Doppler shift and enhanced ion acoustic shoulders. The enhancements are observed at altitudes close to the $\mathrm{F}$ region peak with a small altitude extent of $<2 \mathrm{~km}$. The small altitude extent of the power enhancements is unexpected from the Langmuir turbulence models, which are otherwise used to account for the zero Doppler shift feature in the enhanced spectra.

Acknowledgements. EISCAT is an international association supported by research organisations in China (CRIRP), Finland (SA), Japan (NIPR and STEL), Norway (NFR), Sweden (VR), and the UK (NERC).

Topical Editor K. Hosokawa thanks R. Michell, B. Isham, and one anonymous referee for their help evaluating the paper.

\section{References}

Akbari, H., Semeter, J. L., Dahlgren, H., Diaz, M., Zettergren, M., Strømme, A., Nicolls, M. J., and Heinselman, C.: Anomalous ISR echoes preceding auroral breakup: Evidence for strong Langmuir turbulence, Geophys. Res. Lett., 39, L03102, doi:10.1029/2011GL050288, 2012.

Bahcivan, H. and Cosgrove, R.: Enhanced ion acoustic lines due to strong ion cyclotron wave fields, Ann. Geophys., 26, 2081-2095, doi:10.5194/angeo-26-2081-2008, 2008.

Blixt, E. M., Grydeland, T., Ivchenko, N., Hagfors, T., La Hoz, C., Lanchester, B. S., Løvhaug, U. P., and Trondsen, T. S.: Dynamic rayed aurora and enhanced ion-acoustic radar echoes, Ann. Geophys., 23, 3-11, doi:10.5194/angeo-23-3-2005, 2005.

Buchert, S. C., Tsuda, T., Fujii, R., and Nozawa, S.: The Pedersen current carried by electrons: a non-linear response of the ionosphere to magnetospheric forcing, Ann. Geophys., 26, 28372844, doi:10.5194/angeo-26-2837-2008, 2008. 
Calvert, W.: The auroral plasma cavity, Geophys. Res. Lett., 8, 919921, doi:10.1029/GL008i008p00919, 1981.

Collis, P. N., Häggström, L., Kaila, K., and Rietveld, M. T.: Eiscat radar observations of enhanced incoherent scatter spectra; Their relation to red aurora and field-aligned currents, Geophys. Res. Lett., 18, 1031-1034, doi:10.1029/91GL00848, 1991.

DuBois, D. F., Rose, H. A., and Russell, D.: Coexistence of parametric decay cascades and caviton collapse at subcritical densities, Phys. Rev. Lett., 66, 1970-1973, doi:10.1103/PhysRevLett.66.1970, 1991.

Ekeberg, J., Wannberg, G., Eliasson, L., and Stasiewicz, K.: Ion-acoustic solitary waves and spectrally uniform scattering cross section enhancements, Ann. Geophys., 28, 1299-1306, doi:10.5194/angeo-28-1299-2010, 2010.

Ekeberg, J., Wannberg, G., Eliasson, L., and Häggström, I.: Solitoninduced spectrally uniform ion line power enhancements at the ionospheric F region peak, Earth Planet. Space, 64, 605-611, doi:10.5047/eps.2012.02.005, 2012.

Forme, F. R. E.: Parametric decay of beam-driven Langmuir wave and enhanced ion-acoustic fluctuations in the ionosphere: a weak turbulence approach, Ann. Geophys., 17, 1172-1181, doi:10.1007/s00585-999-1172-4, 1999.

Forme, F. R. E., Fontaine, D., and Wahlund, J. E.: Two Different Types of Enhanced Ion Acoustic Fluctuations Observed in the Upper Ionosphere, J. Geophys. Res., 100, 14625-14636, doi:10.1029/94JA01093, 1995.

Foster, J. C., del Pozo, C., Groves, K., and St. Maurice, J.-P.: Radar observations of the onset of current driven instabilities in the topside ionosphere, Geophys. Res. Lett., 15, 2, 160-163, doi:10.1029/GL015i002p00160, 1988.

Grydeland, T., La Hoz, C., Hagfors, T., Blixt, E. M., Saito, S., Strømme, A., and Brekke, A.: Interferometric observations of filamentary structures associated with plasma instability in the auroral ionosphere, Geophys. Res. Lett., 30, 1338, doi:10.1029/2002GL016362, 2003.

Grydeland, T., Blixt, E. M., Løvhaug, U. P., Hagfors, T., La Hoz, C., and Trondsen, T. S.: Interferometric radar observations of filamented structures due to plasma instabilities and their relation to dynamic auroral rays, Ann. Geophys., 22, 1115-1132, doi:10.5194/angeo-22-1115-2004, 2004.

Guio, P. and Forme, F.: Zakharov simulations of Langmuir turbulence: Effects on the ion-acoustic waves in incoherent scattering, Phys. Plasmas, 13, 122902, doi:10.1063/1.2402145, 2006.

Isham, B., Rietveld, M. T., Guio, P., Forme, F. R. E., Grydeland, T., and Mjølhus, E.: Cavitating Langmuir Turbulence in the Terrestrial Aurora, Phys. Rev. Lett., 108, 105003, doi:10.1103/PhysRevLett.108.105003, 2012.

Lehtinen, M. S. and Huuskonen, A.: General incoherent scatter analysis and GUISDAP, J. Atmos. Terr. Phys., 58, 435-452, doi:10.1016/0021-9169(95)00047-X, 1996.

Michell, R. G. and Samara, M.: High-resolution observations of naturally enhanced ion acoustic lines and accompanying auroral fine structures, J. Geophys. Res., 115, doi:10.1029/2009JA014661, 2010.

Michell, R. G., Lynch, K. A., Heinselman, C. J., and StenbaekNielsen, H. C.: PFISR nightside observations of naturally enhanced ion acoustic lines, and their relation to boundary auroral features, Ann. Geophys., 26, 3623-3639, doi:10.5194/angeo-263623-2008, 2008.
Ogawa, Y., Buchert, S. C., Fujii, R., Nozawa, S., and Forme, F.: Naturally enhanced ion-acoustic lines at high altitudes, Ann. Geophys., 24, 3351-3364, doi:10.5194/angeo-24-3351-2006, 2006.

Ogawa, Y., Buchert, S. C., Häggström, I., Rietveld, M. T., Fujii, R., Nozawa, S., and Miyaoka, H.: On the statistical relation between ion upflow and naturally enhanced ion-acoustic lines observed with the EISCAT Svalbard radar, J. Geophys. Res., 116, A03313, doi:10.1029/2010JA015827, 2011.

Pavan, J., Ziebell, L. F., Yoon, P. H., and Gaelzer, R.: Ionospheric ion-acoustic enhancements by turbulent counterstreaming electron beam-plasma interaction, J. Geophys. Res., 115, A02310, doi:10.1029/2009JA014448, 2010.

Rees, M. H.: Auroral ionization and excitation by incident energetic electrons, Planet. Space Sci., 11, 10, 1209-1218, doi:10.1016/0032-0633(63)90252-6, 1963.

Rietveld, M. T., Isham, B., Grydeland, T., Hoz, C. L., Leyser, T., Honary, F., Ueda, H., Kosch, M., and Hagfors, T.: HF-Pumpinduced parametric instabilities in the auroral E-region, Adv. Space Res., 29, 1363-1368, doi:10.1016/S0273-1177(02)001862, 2002.

Rietveld, M. T., Collis, P. N., and St.-Maurice, J. P.: Naturally Enhanced Ion Acoustic Waves in the Auroral Ionosphere Observed with the EISCAT 933-MHz Radar, J. Geophys. Res., 96, 1929119305, doi:10.1029/91JA01188, 1991.

Robinson, P. A.: Nonlinear wave collapse and strong turbulence, Rev. Mod. Phys., 69, 507-574, doi:10.1103/RevModPhys.69.507, 1997.

Sedgemore-Schulthess, F. and St. Maurice, J.-P.: Naturally Enhanced Ion-Acoustic Spectra And Their Interpretation, Surv. Geophys., 22, 55-92, doi:10.1023/A:1010691026863, 2001.

Steen, ̊., Brändström, U., Gustavsson, B., and Aso, T.: ALIS A Multi-Station Imaging System at High Latitudes with MultiDisciplinary Scientific Objectives, in: European Rocket and Balloon Programmes and Related Research, Vol. 397 of ESA Special Publication, edited by: Kaldeich-Schürmann, B., p. 261, 1997.

Strømme, A., Belyey, V., Grydeland, T., La Hoz, C., Løvhaug, U. P., and Isham, B.: Evidence of naturally occurring wave-wave interactions in the polar ionosphere and its relation to naturally enhanced ion acoustic lines, Geophys. Res. Lett., 32, L05103, doi:10.1029/2004GL020239, 2005.

Sullivan, J. M., Lockwood, M., Lanchester, B. S., Kontar, E. P., Ivchenko, N., Dahlgren, H., and Whiter, D. K.: An optical study of multiple NEIAL events driven by low energy electron precipitation, Ann. Geophys., 26, 2435-2447, doi:10.5194/angeo-262435-2008, 2008.

Zakharov, V. E.: Collapse of Langmuir waves, Sov. Phys. JETP, 35, 908-914, 1972.

Ziebell, L. F., Yoon, P. H., Pavan, J., and Gaelzer, R.: Ionacoustic enhancements generated by beam-plasma instability in an auroral cavity, J. Geophys. Res., 116, A03320, doi:10.1029/2010JA016147, 2011. 